\title{
Performance Evaluation of a Dedicated Camera Suitable for Dynamic Radiopharmaceuticals Evaluation in Small Animals
}

George Loudos, Member, IEEE, Stan Majewski, Member, IEEE, Randy Wojcik, Andrew Weisenberger, Nicolas Sakellios, Konstantina Nikita, Member, IEEE, Nikolaos Uzunoglu, Member, IEEE, Penelope Bouziotis, Stavros Xanthopoulos, and Alexandra Varvarigou

\begin{abstract}
As the result of a collaboration between the Detector and Imaging Group of Thomas Jefferson National Accelerator Facility (US), the Institute of Radioisotopes and Radiodiagnostic Products (IRRP) of N.C.S.R. "Demokritos" and the Biomedical Simulations and Imaging Applications Laboratory (BIOSIM) of National Technical University of Athens (Greece), a mouse sized camera optimized for $\mathrm{Tc}^{99 \mathrm{~m}}$ imaging was developed. The detector was built in Jefferson Lab and transferred to Greece, where it was evaluated with phantoms and small animals. The system will be used initially for planar dynamic studies in small animals, in order to assess the performance of new radiolabeled biomolecules for oncological studies. The active area of the detector is approximately $48 \mathrm{~mm} \times 96 \mathrm{~mm}$. It is based on two flat-panel Hamamatsu H8500 position sensitive photomultiplier tubes (PSPMT), a pixelated $\mathrm{NaI}(\mathrm{Tl})$ scintillator and a high resolution lead parallel-hole collimator. The system was developed to optimize both sensitivity and resolution for in vivo imaging of small animals injected with technetium compounds. The results of system evaluation in planar mode with phantoms are reported. Results are presented for in vivo dynamic studies of mice injected with $>100 \mu \mathrm{Ci}$ of two conventional and novel radiopharmaceuticals, namely $\mathrm{Tc}^{99 \mathrm{~m}}$ -MDP and $\mathrm{Tc}^{99 \mathrm{~m}}$-Bombesin.
\end{abstract}

Index Terms-Dedicated gamma camera, position sensitive photomultiplier tubes, radiopharmaceuticals, small animal imaging.

\section{INTRODUCTION}

D YNAMIC planar imaging is a very useful tool in order to assess the behavior of radiolabeled molecules in small animals. In this type of imaging it is essential that the detector produces a seamless image of the entire animal at sufficiently high resolution and sensitivity. A number of dedicated imagers have been presented over the past years using a variety of approaches for scintigraphic and/or SPECT studies [1]-[7]. Many of these systems are based on Position Sensitive Photomultiplier Tubes (PSPMTs), since they meet these requirements. Since the

Manuscript received February 7, 2006; revised January 4, 2007.

G. Loudos, N. Sakellios, K. Nikita, and N. Uzunoglu are with the School of Electrical and Computer Engineering, National Technical University of Athens, Iroon Polytechniou 9, 15780 Zografos, Greece (e-mail: gloudos@bioacademy.gr; snicol@biosim.ntua.gr; knikita@cc.ece.ntua.gr; nnap@otener.gr).

S. Majewski, R. Wojcik, and A. Weisenberger are with Thomas Jefferson National Accelerator Facility, Newport News, VA 23606 USA (e-mail: stan@jlab. org; rfwojcik@rayvisions.com; drew@jlab.org).

P. Bouziotis, S. Xanthopoulos, and A. Varvarigou are with the Institute of Radioisotopes-Radiodiagnostic Products, National Center for Scientific Research "Demokritos," 15310 Athens, Greece (e-mail: pennybil@yahoo.gr; avar@rrp. demokritos.gr).

Digital Object Identifier 10.1109/TNS.2007.896212

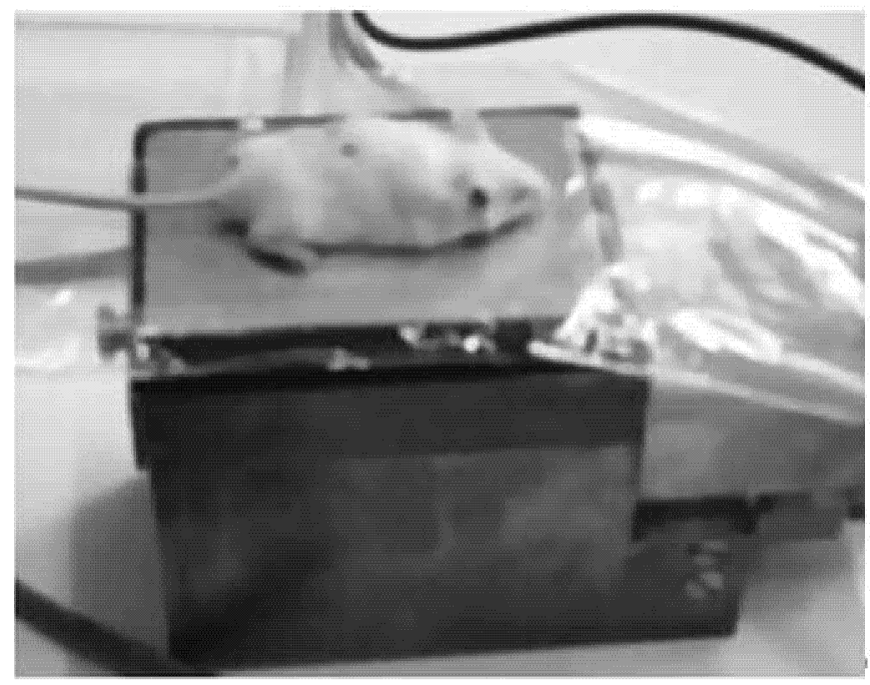

Fig. 1. Detector head, with an anaesthetized mouse placed on it, in a typical imaging position.

first PSPMTs were circular, their size limited the field of view (FOV). The R3292 PSPMT (5 inches in diameter) has been a suitable detector for the construction of systems that can image the entire mouse in a single view [8]-[10]. The commercial availability of a new generation of square PSPMTs allowed the construction of larger FOV cameras based on the combination of two or more PSPMTs, while methods for recovering signals in the dead area between the PSPMTs were developed [11].

A very promising component for cameras based on more than one square tube is the $\mathrm{H} 8500$, which was well tested by several groups [12]-[16] and has already been used in small animal imagers [17]-[20]. One of the techniques used, reported by Popov et al. [11], was applied in the work described here in order to retain detector sensitivity across the $3 \mathrm{~mm}$ gap between the two tubes' photocathodes at the junction between the pair of PSPMTs.

In our design, a pair of the H8500 tubes was combined and attached to a pixelated $\mathrm{NaI}(\mathrm{Tl})$ crystal array with a high resolution lead parallel-hole collimator designed for use with compounds tagged with $\mathrm{Tc}^{99 \mathrm{~m}}$. The detector package is enclosed in tungsten housing suitable for mounting on a rotating gantry for future use SPECT imaging. The gamma camera is presented in Fig. 1 with an anaesthetized mouse placed on it, in a typical imaging position. 
In the present work we describe the components of this system and we report the measured spatial and energy resolution and sensitivity in planar mode. The results of capillary imaging and the planar image of mice injected with $\mathrm{Tc}^{99 \mathrm{~m}}$ -MDP demonstrate the high resolution of the system. Finally, a "proof of principle" series of kinetic studies of the uptake of $\mathrm{Tc}^{99 \mathrm{~m}}$-Bombesin derivatives, show that the developed system is an efficient imager that will be useful to evaluate newly developed radiopharmaceuticals in small animals.

Although the studies are mainly focused on the use of the imager the challenges of this work were i) the smallest useful full mouse size imager used in a planar mode, ii) economy and simplicity in planar mode including SPECT potential for a fraction of a cost of commercial systems, iii) the development of a novel detector with the best attainable resolution in a pixelated $\mathrm{NaI}(\mathrm{Tl})$ array, not currently commercially available, iv) very reliable inexpensive technology as opposed to a photodiode-based readout or Cadmium Zinc Telluride (CZT), v) modular design (two PMTs read separately) that can be easily extended to larger sizes and finally vi) optimized optical coupling between the PMTs by also using a rather simple readout.

\section{MATERIALS}

\section{A. Hamamatsu H8500 PSPMTs}

The H8500 is a square, flat-panel PSPMT with external dimensions of $52 \mathrm{~mm} \times 52 \mathrm{~mm} \times 34 \mathrm{~mm}$ thick. It is a 12 stage tube with a gain of 106 and an $8 \times 8$ array of anode pads. The optimal high voltage (HV) for system operation was found to be $795 \mathrm{~V}$ for Tc $99 \mathrm{~m}$. HV was selected to be low to avoid noisy operation but high enough to assure good signal to noise ratio (SNR).

\section{B. NaI(Tl) Pixelated Scintillation Crystal}

The scintillator employed in this detector is of pixelated $\mathrm{NaI}(\mathrm{Tl})$ and is manufactured by Bicron-St. Gobain (Bicron-St. Gobain Inc. Cleveland, OH, and Paris, France). The crystal array has an active area of $97.4 \mathrm{~mm} \times 44.8 \mathrm{~mm}$ to match the active region of the pair of adjoined phototubes. The pixel dimensions are $1 \mathrm{~mm} \times 1 \mathrm{~mm} \times 5 \mathrm{~mm}$ with a pitch of $1.2 \mathrm{~mm}$. The $5 \mathrm{~mm}$ thickness is the maximum attainable with $1 \mathrm{~mm}$ pixels. The array is viewed through a $3 \mathrm{~mm}$ glass window and encapsulation is completed by an aluminum cover $50 \mu \mathrm{m}$ thick. Owing to the difficulty in cutting and sealing arrays of such small pixels of $\mathrm{NaI}(\mathrm{Tl})$, the manufacturer made a protective outer perimeter of $2 \mathrm{~mm}$ pixels for added integrity of the array.

\section{The Parallel-Hole Collimator}

For initial studies of this system a high resolution parallelhole collimator was employed. The collimator, fabricated by Precise Corporation (Precise Corporation, Caryville, TN 37714 USA), is $25 \mathrm{~mm}$ thick and has an active area $52 \mathrm{~mm} \times 105 \mathrm{~mm}$. The collimator measures $58 \mathrm{~mm} \times 122 \mathrm{~mm}$ in area owing to the presence of a frame on three sides of the active rectangular area. It has hexagonal cells $1.2 \mathrm{~mm}$ from flat to flat sides separated by $0.2 \mathrm{~mm}$ thick septum walls. The collimator is manufactured in a way that a single septum exists along its long and a double septum exists along its short dimension.

\section{Assembly of the Detector}

A box measuring $140 \mathrm{~mm} \times 82 \mathrm{~mm} \times 107 \mathrm{~mm}$ deep machined of $8 \mathrm{~mm}$ thick tungsten (W) is used to contain the detector system. The front face entrance window is $0.5 \mathrm{~mm}$ thick aluminum. The presence of aluminium affects sensitivity and scatter by only a factor of $\sim 0.5 \%$, as was shown by Monte Carlo simulations using GATE [21]. A mounting bracket extends from the narrow end for mounting on a gantry. Inside the Al box, the two H8500 PSPMTs are gently taped together and then taped against the glass face of the $\mathrm{NaI}(\mathrm{Tl})$ scintillator. Optical grease (RX-688 Optical Coupling Grease, Rexon Inc., Beachwood, OH, 44122) was used for optical coupling of the PSPMTs to the scintillation array window. The collimator is mounted in a tungsten frame and can be easily slid on and off the housing on a built-in rail. It is held in place with a thumb nut which threads onto a tungsten screw mounted in the housing. The combination of the two PSPMTs and the NaI(Tl) array are gently pressed against the back of the aluminum window within the box.

\section{E. Anode Readout}

Although each of the PSPMTs has 64 anode pads in an 8 by 8 array, the signals are read out from each tube via a matrix of resistors arranged to allow the $\mathrm{X}$ and the $\mathrm{Y}$ signals from one tube to be represented by 8 amplified signal lines for each dimension per PMT [12]. The 16 signals on each PMT are then connected to a subtractive resistive readout which reduces the number of signals to just four per PMT plus one sum signal used for triggering. The sum is sent to a fast constant fraction discriminator and the eight signals are collected using two $\mathrm{Na}-$ tional Instruments PCI6110 ADCs which have 4 channels each. The Kmax-based (Sparrow Corp., Port Orange, FL, 32128) data acquisition algorithm calculates the digitized signals from both PSPMTs to permit a single continuous active detector surface in one of the detection coordinates [8]. As the two PSPMTs do not have identical gain at the same bias voltage, they are operated at different high voltages and this is currently achieved with a single HV power supply and a resistive divider.

\section{METHODS}

\section{A. System Calibration}

In order to create system's look up table (LUT) for crystal identification, flood irradiation is necessary. A point-like Tc99 $\mathrm{m}$ source was used, placed $50 \mathrm{~cm}$ away from the detector with the collimator removed. Using custom made software and the raw image, the area around each crystal cell is defined and the crystal pixel LUT is generated; the software calculates the center of gravity (COG) for all crystal cells in the raw image, by finding all locally hot pixels. Then by taking into account the neighbouring COG's it adjusts the optimal number of pixels that belong to this crystal element. The collimator was used again and a flood source covering the entire field of view was used. By summing all the pixels of the raw image the summed flood image is generated. This flood image is used for calibration of acquired images and it includes both crystal and collimator response. 


\section{B. Spatial Resolution}

The system's spatial resolution was measured using a thin capillary (1.1 mm inner diameter and $8 \mathrm{~cm}$ long) filled with a Tc99 m solution. The capillary was placed with a slight rotation ( $\sim 3$ crystal pixels wide) and three profiles were averaged. Since the dimensions of the capillary are comparable to the crystal pixels, using this technique we avoid the effects of capillary positioning over one (best case scenario) or two (worst case scenario) rows of crystal pixels. In order to estimate spatial resolution thirteen measurements were taken at 0-12 cm distance from the detector surface.

The system's high spatial resolution was assessed by placing three capillaries side-by-side, right on the detector surface. The middle one was empty, while the other two were filled with a Tc99 m solution. The center-to-center distance between the two filled capillaries is $3 \mathrm{~mm}$ and the "dead area" between them is $1.9 \mathrm{~mm}$. Moreover four capillaries were filled with Tc99 m solution and with relative activities of approximately $100 \%, 50 \%$, $25 \%$, and $12.5 \%$. The phantom was imaged for 10 secs and $\sim 10000$ counts were acquired.

\section{Energy Response}

The system's energy response was measured with a Tc99 m point-like source with the collimator removed. Regions of interest (ROIs) were drawn at the area of the two PSPMTs and the area between them, in order to measure differences in energy response.

\section{Sensitivity}

Sensitivity was measured as a function of source to detector distance, with a flood source containing $200 \mathrm{ml}$ of a $0.5 \mathrm{mCi}$ Tc99 m solution. The dimensions of the phantom were greater than the detector's field of view. The phantom was placed at 0 , 5 and $10 \mathrm{~cm}$ distance from the detector surface. In order to test the system's response as a function of a source activity, a flood source with dimensions $3 \mathrm{~cm} \times 4 \mathrm{~cm}$ was placed on the camera. The source was filled with a Tc99 $\mathrm{m}$ solution with activities ranging from $0.2 \mathrm{mCi}$ to $10 \mathrm{mCi}$.

\section{E. Static Image of a Mouse Injected With Tc99 m-MDP}

Initially the system was tested using a normal mouse injected intravenously into the tail vein with $1 \mathrm{mCi}$ of Tc99 m-MDP, a standard derivative, suitable for bone imaging. The mouse was anesthetized by the subcutaneous injection of $1.0 \mathrm{~g}$ of 2,2,2tribromomethanol in $1.0 \mathrm{ml}$ of 2-methyl-2-butanol, at a dose of 100 mul per $30 \mathrm{~g}$ body weight. The mouse was placed on the camera $20 \mathrm{~min}$ after the Tc99 m-MDP injection and data were acquired for six minutes.

\section{F. Kinetic Studies With Tc99 m-Bombesin Derivatives}

Currently the system is being used for the evaluation of novel radiopharmaceuticals. Kinetic studies have been carried out in tumor bearing nude mice using Tc99 m-Bombesin derivatives and injected doses vary between $100 \mu \mathrm{Ci}$ and $500 \mu \mathrm{Ci}$. Mice are anaesthetized and placed onto the camera, right after the injection. We present five sequential images obtained between 10 and 20 minutes after the injection of $200 \mu \mathrm{Ci}$ Tc99 m-Bombesin, when maximum concentration in the tumor is observed.
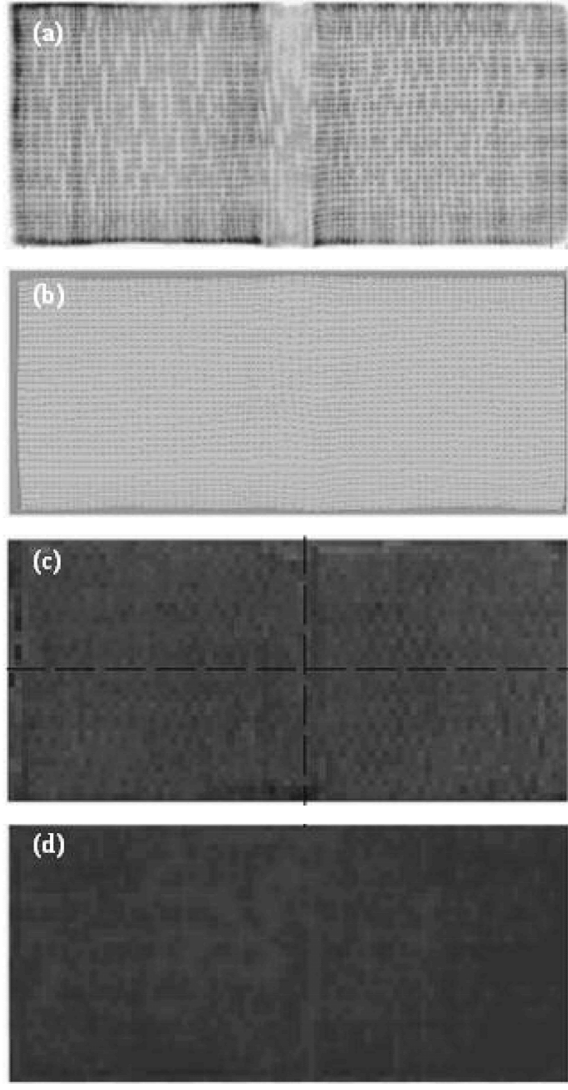

Fig. 2. (a) A raw image of the detector obtained, with a point-like $\mathrm{Tc}^{99 \mathrm{~m}}$ source, placed $50 \mathrm{~cm}$ away from the detector, with the collimator removed. (b) Crystal pixel lookup table. (c) Summed flood image. The dashed lines indicate the position of the profiles that are presented in Fig. 3. (d) Image of a uniform source covering the entire field of view and corrected using the flood image of (c).

\section{RESULTS}

\section{A. Phantom Studies}

A raw image of the detector [Fig. 2(a)] shows the presence of the transition region at the gap between the two phototubes. The obtained LUT is shown in Fig. 2(b) and the summed non-corrected flood image in Fig. 2(c). Finally the image of a uniform source covering the entire field of view and corrected using the summed image is shown in Fig. 2(d).

Some hexagonal effects visualized, mainly on the right part of the image, may be the result of collimator holes and pixelated crystal misalignment. This effect does not appear in the flood corrected images, as it can be seen in Fig. 2(d). No detection efficiency loss or visible deterioration of performance was observed in the gap region as it can be seen in the summed flood image [Fig. 2(c)] and in the two typical horizontal and vertical profiles in Fig. 3.

In addition, profiles demonstrate the relatively uniform response in both directions of the field of view, even in the noncorrected flood image.

In Fig. 4 the image of a thin capillary and the profiles used to measure spatial resolution are shown.

Average resolution was $\sim 1.6 \mathrm{~mm}$ on the collimator surface and increased to $\sim 4.1 \mathrm{~mm}, 12 \mathrm{~cm}$ from the collimator surface. Spatial resolution curve is shown in Fig. 5. 

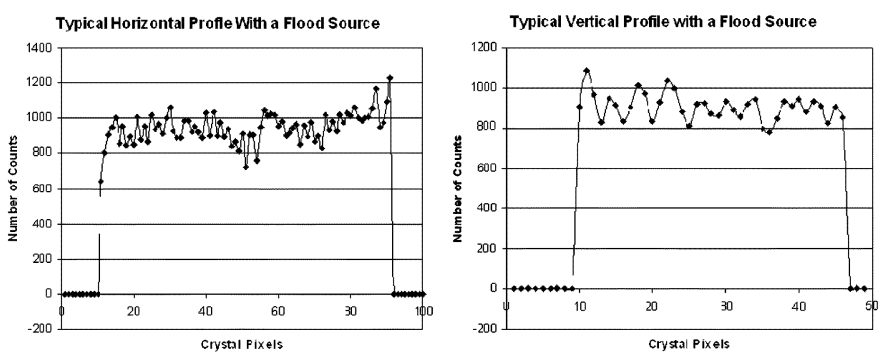

Fig. 3. Typical horizontal and vertical profiles in the center of the flood (noncorrected) image, showing the detector uniformity across the entire field of view and in the insensitive region between the two PSPMTs.

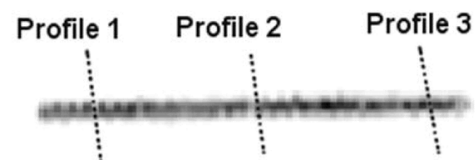

Fig. 4. Image of a thin capillary ( $1.1 \mathrm{~mm}$ inner diameter, $8 \mathrm{~cm}$ long) filled with a $\mathrm{Tc}^{99 \mathrm{~m}}$ solution. Positioning of the three profiles that are averaged, in order to calculate spatial resolution as a function of source to detector distance. In order to determine FWHM, linear interpolation was used in the profiles.

\section{Spatial Resolution}

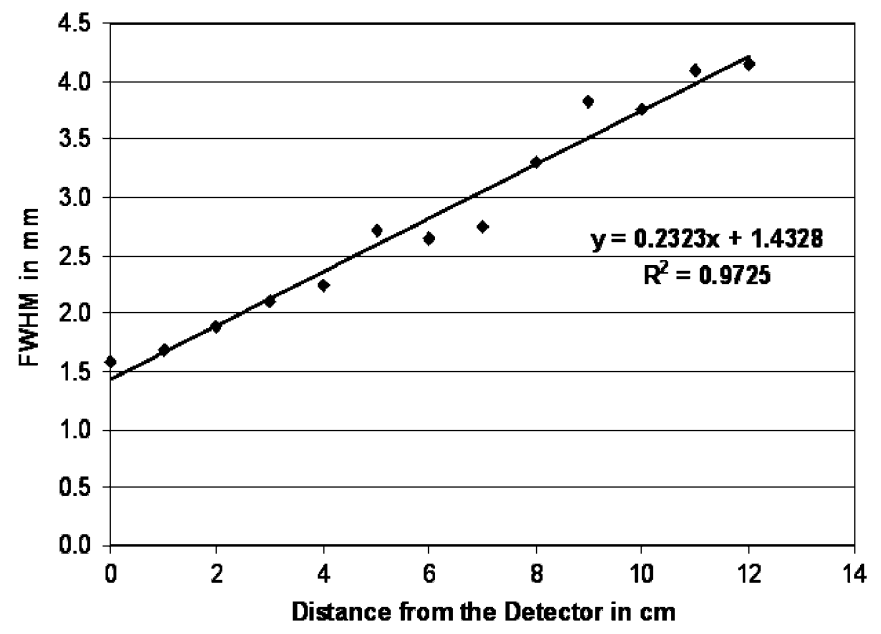

Fig. 5. Spatial resolution as a function of source to detector distance, using the capillary and by applying the methods of Fig. 4 .

In addition, profiles demonstrate the relatively uniform response in both directions of the field of view, even in the noncorrected flood image.

In Fig. 4 the image of a thin capillary and the profiles used to measure spatial resolution are shown.

Average resolution was $\sim 1.6 \mathrm{~mm}$ on the collimator surface and increased to $\sim 4.1 \mathrm{~mm}, 12 \mathrm{~cm}$ from the collimator surface. Spatial resolution curve is shown in Fig. 5.

The results from capillary imaging are shown in Fig. 6. As it can be observed in the image and the corresponding typical profile, the system is able to separate the two capillaries.

In Fig. 7 the results from measurements of the system's energy response are shown. The total energy spectrum is affected by the non-uniform response of the PMTs, as well as the loss of signal in the area between them (Fig. 7 middle). However, by calculating the energy spectrum of each crystal cell and by normalizing them using the peak shift method, an improved total
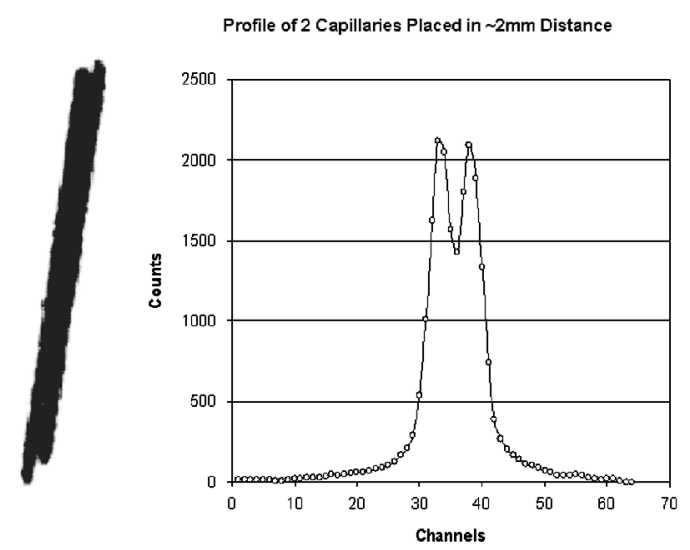

Fig. 6. Image and typical profile of two capillaries (1.1 $\mathrm{mm}$ inner diameter) filled with a $\mathrm{Tc}^{99 \mathrm{~m}}$ solution and separated by a similar empty capillary.

normalized energy spectrum is obtained, as it can be seen in the bottom image of Fig. 7. The energy resolution was measured and found to be $\sim 15.6 \%$ for $\mathrm{Tc}^{99 \mathrm{~m}}$.

In Fig. 8 the sensitivity as a function of distance from the collimator is shown; only a small decrease in sensitivity is observed. The sensitivity was $\sim 130$ counts $/ \mathrm{min} / \mu$ Ci. Since in animal studies mice are injected with $100-1000 \mu \mathrm{Ci}$, $13000-130000$ counts per minute are expected, which is a satisfactory number, in order to perform kinetic studies.

In Fig. 9 the system's response measured in counts $/ \mathrm{min} / \mathrm{cm} 2$ is presented.

A good linear response is observed and a satisfactory number of $\sim 5000$ counts $/ \mathrm{min} / \mathrm{mCi} / \mathrm{cm}^{2}$ is achieved. There is also good agreement between data in Figs. 8 and 9. The entire active field of view (excluding septa between crystal pixels) is $\sim 30 \mathrm{~cm}^{2}$. Thus the sensitivity of $\sim 130$ counts $/ \mathrm{min} / \mu \mathrm{Ci}$ corresponds to $\sim 4333$ counts $/ \mathrm{min} / \mathrm{mCi} / \mathrm{cm}^{2}$, which is in good agreement with the 4124 counts $/ \mathrm{min} / \mathrm{mCi} / \mathrm{cm}^{2}$ that we have measured using the $3 \mathrm{~cm} \times 4 \mathrm{~cm} \mathrm{Tc}^{99 \mathrm{~m}}$ source (third point in Fig. 9).

Fig. 10 shows the results from imaging four capillaries filled with $\mathrm{Tc}^{99 \mathrm{~m}}$ solutions with relative activities of approximately $100 \%, 50 \%, 25 \%$ and $12.5 \%$. A very short scan was carried out and only $\sim 10000$ counts were collected. As it can be seen in the image and the corresponding typical profile, activity variations were detected with good approximation. Some gaps in the capillaries are due to small air bubbles when filling the capillaries. Such gaps have not been observed when imaging more complicated phantoms or mice.

\section{B. Small Animal Studies}

Fig. 11 shows a static image of a normal mouse injected with $^{99 \mathrm{~m}}$ Tc-MDP. Since the mouse was alive and there was increased concentration in the kidneys and the bladder, a threshold was applied to high intensity values (more than 800 counts). Evident are the chest bones, as well as the "gap" in the lungs' position. The resolution of the detector and its applicability to the imaging of mice is clearly demonstrated in this image.

In Fig. 12 the results of the experiment using a ${ }^{99 \mathrm{~m}} \mathrm{Tc}-$ Bombesin derivative are presented. Five intervals of 2 minutes are shown and a threshold to high intensity values (more than 


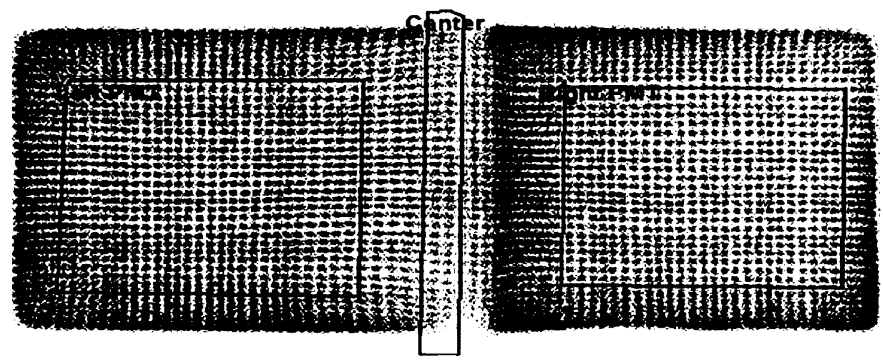

Energy spectra in the three Rols

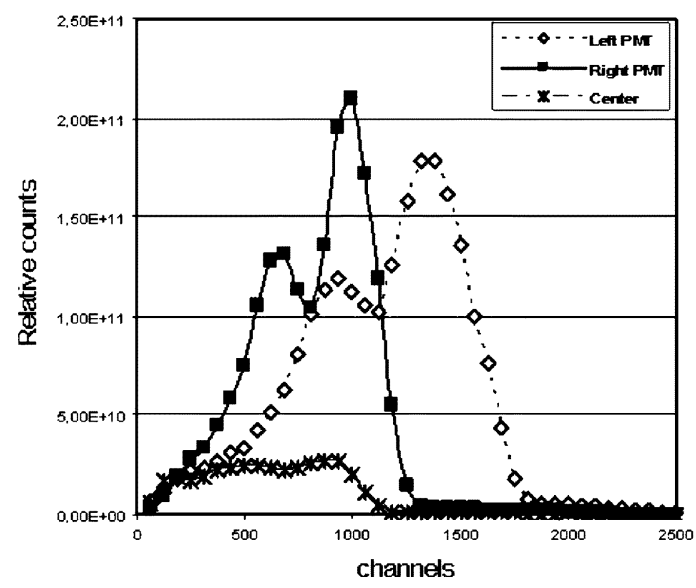

Normalized and total energy spectra

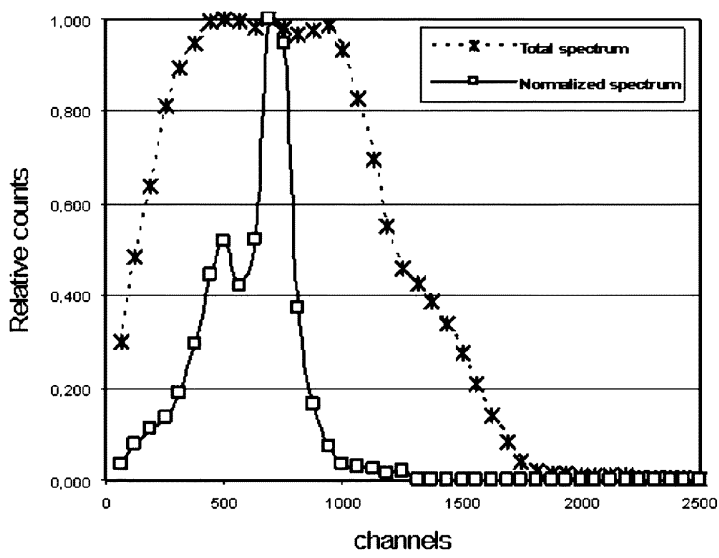

Fig. 7. (Top) ROIs in the two PMTs and the area between them. (Middle) Energy spectrum in the three ROIs. (Bottom) Total uncorrected and normalized energy spectrum.

140 counts) was applied. Approximately 150000 counts in each frame were collected.

Since Bombesin derivatives are now in the design and evaluation stage we still measure relatively low uptake in the tumor and masking is necessary. In addition, as stated before, there is increased uptake in bowels and bladder. Thus, beside radiobiological parameters the experimental protocols are being investigated, including imaging of fasted or thirsty animals, in order to reduce background. From this example it can be demonstrated that the system allows the performance of kinetic studies in small mice, in order to assess novel radiopharmaceuticals. Such image series can be used in order to draw time-activity curves, although more precise results should be derived from SPECT imaging, which is our next step.

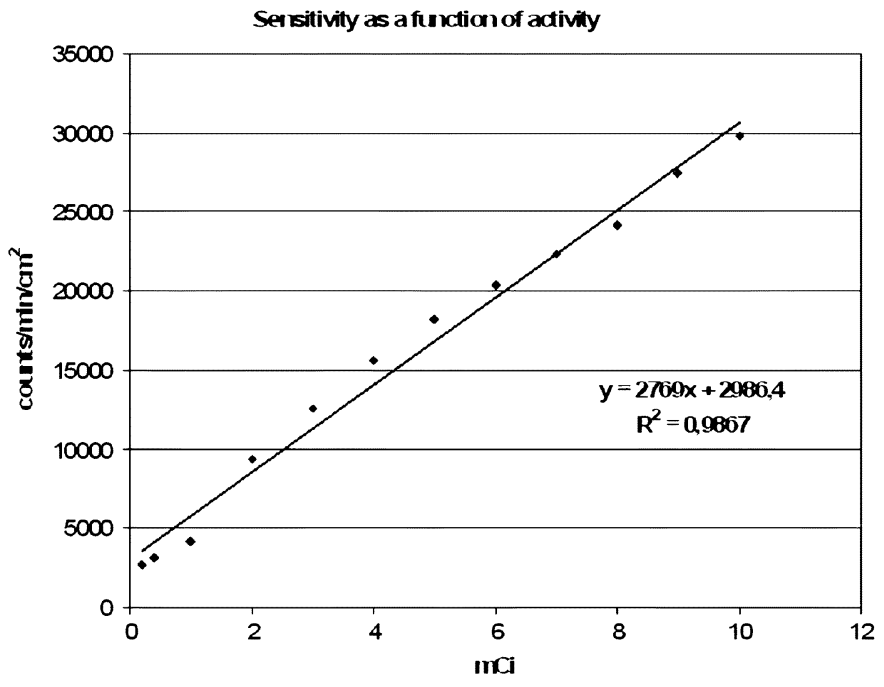

Fig. 8. Sensitivity, as a function of source to detector distance. A flood source containing $200 \mathrm{ml}$ of a $0.5 \mathrm{mCi} \mathrm{Tc}^{99 \mathrm{~m}}$ solution was used.

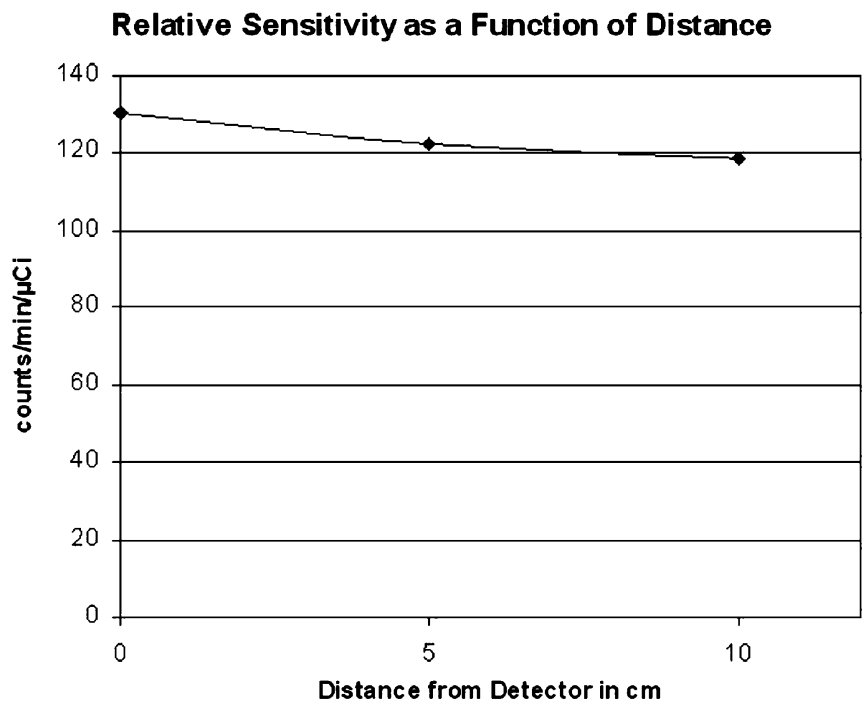

Fig. 9. System's response for varying source activities, measured in counts/ $\mathrm{min} / \mathrm{cm}^{2}$ for a $3 \mathrm{~cm} \times 4 \mathrm{~cm} \mathrm{Tc} \mathrm{c}^{99 \mathrm{~m}}$ source.
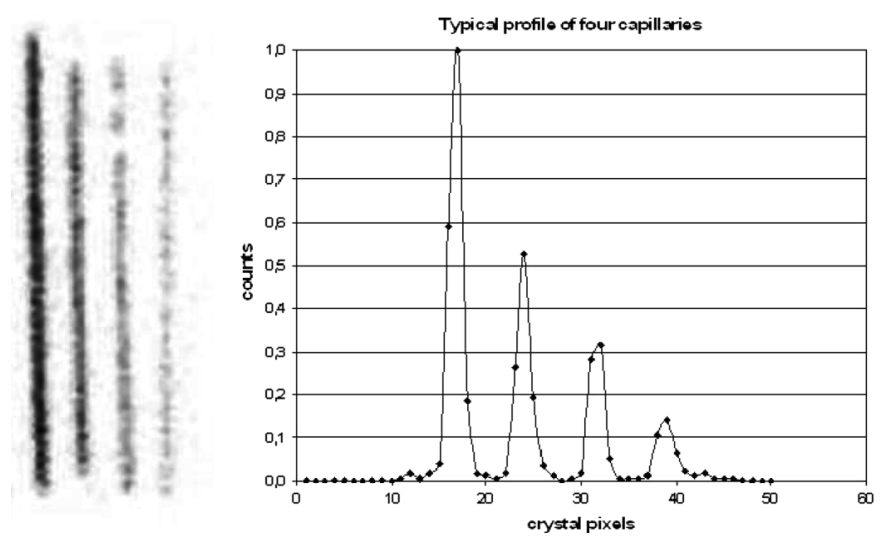

Fig. 10. Image and typical profile of four capillaries (1.1 $\mathrm{mm}$ inner diameter) filled with four $\mathrm{Tc}^{99 \mathrm{~m}}$ solutions with relative activities $100 \%, 50 \%, 25 \%$ and $12.5 \%$. 


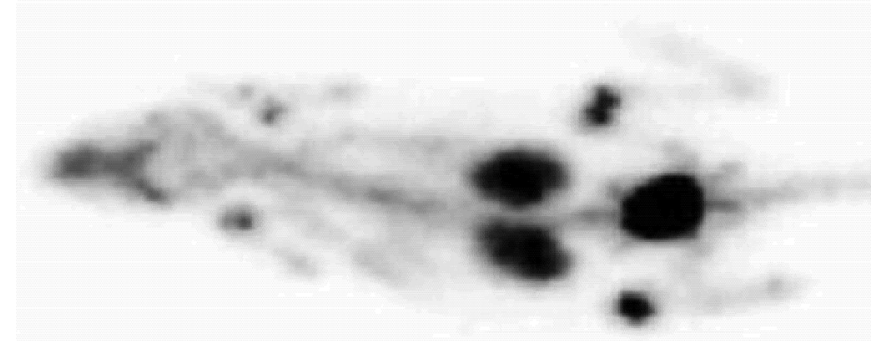

Fig. 11. An anaesthetized normal mouse injected with $1 \mathrm{mCi} \mathrm{Tc}^{99 \mathrm{~m}}-\mathrm{MDP}$ and imaged for six minutes. An upper threshold to image counts was applied to bladder and kidneys. Mouse bones are clearly visualized.

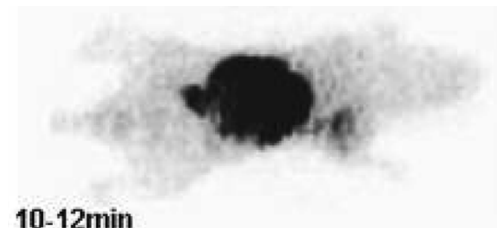

10-12min

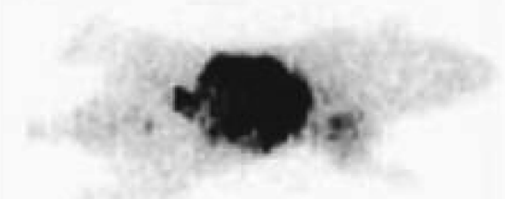

12-14min

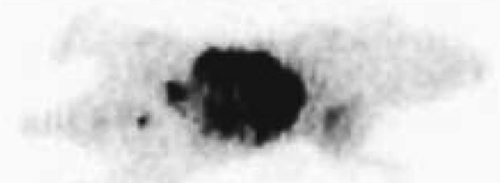

14-16min

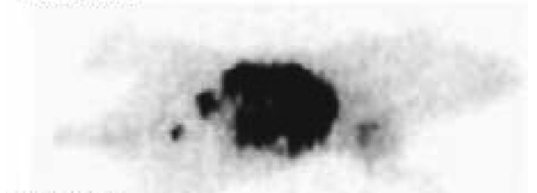

16-18min

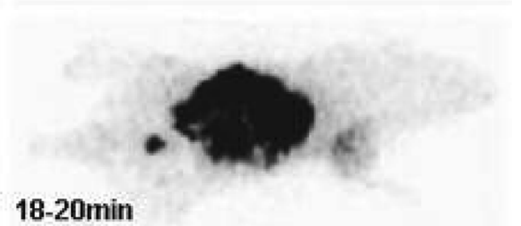

Fig. 12. Five sequential images of a small tumor bearing nude mouse injected with $200 \mu \mathrm{Ci}$ of a $\mathrm{Tc}^{99 \mathrm{~m}}$-Bombesin derivative. The tumor was implanted close to the right shoulder. A threshold was applied to high image values, in order to allow tumor visualization. Two minutes intervals have been used.

\section{DisCuSSION AND Future GoALS}

The presented results show that the system described here is suitable for the performance of dynamic studies in small animals, based on its size, resolution and sensitivity. Currently, a parallel-hole collimator was used. The camera is being used in order to assess new radiolabeled derivatives for tumor imaging and angiogenesis visualization such as $\mathrm{Tc}^{99 \mathrm{~m}}$-labeled Bombesin derivatives, $\mathrm{Tc}^{99 \mathrm{~m}}$-labeled RGD derivatives to image experimental breast cancer, etc. The system is optimized in order to assess the performance of $\mathrm{Tc}^{99 \mathrm{~m}}$-based derivatives, but can be also applied to imaging other isotopes such as
$\mathrm{I}^{125}, \mathrm{Sm}^{153}$ and $\mathrm{Lu}^{177}$, with proper calibration and collimator replacement.

Comparing this camera to other similar existing systems it provides the best spatial resolution by using pixelated $\mathrm{NaI}(\mathrm{Tl})$ with a $1.2 \mathrm{~mm}$ crystal pitch. In addition, the design of the system was optimized in order to decode such small crystal pixels in the gap region between PMTs using simple readout electronics. Bradley et al., [18] have built a mouse-sized camera, equipped with a parallel hole collimator, optimized for SPECT imaging using $\mathrm{I}^{125}$ derivatives. In this system spatial resolution of $2.3 \mathrm{~mm}$ FWHM is measured, while in our design it is $\sim 1.6 \mathrm{~mm}$. Cinti et al., [19] have used an H8500 detector, equipped with a high resolution $\mathrm{Cu}$-Be parallel hole collimator. Spatial resolution was better than $2 \mathrm{~mm}$ and energy resolution 10\%; however a multianode readout was used compared with the simple readout used here. Olcot et al., [16] using a single $\mathrm{H} 8500$ detector and charge division, have found value of $\sim 1.8 \mathrm{~mm}$ and $\sim 13 \%$ for spatial and energy resolution respectively, while the problem of the gap between the two tubes was not addressed. The obtained results are in good agreement with similar studies using the circular tubes R2486 [3] and R3292 [22].

The next step will be to evaluate the system's performance in small animal imaging, using a $1 \mathrm{~mm}$ pinhole collimator, mainly for the magnification of small organs [19], [23]. The end goal of the collaboration is the development of a full SPECT/CT system based on designs that have been presented [24]. We plan to start by using one detector head, thus it is important to discuss system limitations.

In the study presented in Fig. 12, 150 000 counts were acquired in 2 minutes, when $200 \mu \mathrm{Ci}$ were injected into the mouse. From the studies that we have already performed, we believe that sufficient information is obtained with shorter scans e.g., 1 min and $\sim 75000$ counts. Thus, in order to acquire a typical number of projections (60 to 72) a scan of more than one hour is necessary, which does not allow the performance of dynamic SPECT studies, but only tomographic imaging of sacrificed animals. However, in a number of published SPECT studies much higher activities have been used, such as $4 \mathrm{mCi}$ [25] or even $24 \mathrm{mCi}$ [26], which would substantially reduce acquisition time by a factor of 20 or 120 . We have demonstrated the linear response of our system up to $10 \mathrm{mCi}$ and we can determine its saturation limits. Using such high activities SPECT kinetic studies are in principle feasible with this camera, but it is necessary to carefully study the effects of increased radiation in small animals, since increased doses may alter the results of such studies [1]. We plan to investigate experimentally systems limitations in SPECT mode. Currently, we have simulated the system with GATE [21] and we are carrying out simulations with MOBY mouse phantom [27], in order to study acquisition parameters in SPECT [28].

\section{REFERENCES}

[1] P. D. Acton and H. F. Kung, "Small animal imaging with high resolution single photon emission tomography," Nucl. Med. Biol., vol. 30, pp. 889-95, 2003.

[2] G. A. Kastis, "Gamma-ray imaging using a CdZnTe pixel array and a high-resolution, parallel-hole collimator," IEEE Trans. Nucl. Sci., vol. 47, no. 6, pp. 1923-1927, Dec. 2000. 
[3] G. K. Loudos, "A 3D high resolution gamma-camera for radiopharmaceutical studies with small animals," Appl. Radiot. Isot., vol. 58, pp. 501-508, 2003.

[4] K. Ogawa, T. Kawada, K. Nakamura, A. Kubo, and T. Ichihara, "Ultra high resolution pinhole SPECT for small animal study," IEEE Trans. Nucl. Sci., vol. 45, no. 6, pp. 3122-3126, Dec. 1998.

[5] Z. Liu, "Quantitative analysis of acute myocardial infarct in rat hearts with ischemia-reperfusion using a high-resolution stationary SPECT system," J. Nucl. Med., vol. 43, pp. 933-939, 2002.

[6] D. P. McElroy, "Performance evaluation of A-SPECT: A high resolution desktop pinhole SPECT system for imaging small animals," IEEE Trans. Nucl. Sci., vol. 49, no. 5, pp. 2139-2147, Oct. 2002.

[7] N. U. Schramm, G. Ebel, U. Engeland, T. Schurrat, M. Béhé, and T. M. Behr, "High-resolution SPECT using multipinhole collimation," IEEE Trans. Nucl. Sci., vol. 50, no. 3, pp. 315-320, Jun. 2003.

[8] R. Wojcik, S. Majewski, B. Kross, D. Steinbach, and A. G. I. Weisenberger, "High spatial resolution gamma imaging detector based on 5", diameter R3292 Hamamatsu PSPMT," IEEE Trans. Nucl. Sci., vol. 45, no. 3, pp. 487-491, Jun. 1998.

[9] N. Schramm, A. Wirrwar, F. Sonnenberg, and H. Halling, "Compact high resolution detector for small animal SPECT," IEEE Trans. Nucl. Sci., vol. 41, no. 3, pp. 1163-1167, Jun. 2000.

[10] T. Y. Song, Y. Choi, and Y. H. Chung, "Performance amelioration for small animal SPECT using optimized pinhole collimator and image correction technique," in Proc. IEEE Nuclear Science Symp. and Medical Imaging Conf. Rec., Oct. 2004, vol. 6, pp. 3458-3462.

[11] V. Popov, S. Majewski, A. G. Weisenberger, and R. Wojcik, "Analog readout system with charge division type output," in Proc. IEEE Nuclear Science Symp. and Medical Imaging Conf. Rec., San Diego, CA, Nov. 2001, pp. 1937-1940.

[12] R. Pani, "Factors affecting flat panel PMT calibration for gamma ray imaging," in Proc. IEEE Nuclear Science Symp. and Medical Imaging Conf. Rec., Nov. 2002, vol. 2, pp. 671-675.

[13] R. Engels, U. Clemens, G. Kemmerling, and J. Schelten, "High spatial resolution scintillation detector based on the H8500 photomultiplier," in Proc. IEEE Nuclear Science Symp. and Medical Imaging Conf. Rec., Oct. 2003, vol. 1, pp. 692-695.

[14] S. Korpar, "Tests of a proximity focusing RICH with aerogel radiator and flat-panel multianode PMTs (H8500)," in Proc. IEEE Nuclear Science Symp. and Medical Imaging Conf. Rec., Oct. 2003, vol. 1, pp. $147-150$.

[15] C. Lerche, "Depth of interaction measurement in gamma ray imaging detectors with continuous scintillation crystals," in Proc. IEEE Nuclear Science Symp. and Medical Imaging Conf. Rec., Oct. 2003, vol. 3, pp. 2169-2173.
[16] P. D. Olcott, F. Habte, C. S. Levin, and A. M. Foudray, "Characterization of performance of a miniature, high sensitivity gamma ray camera," in Proc. IEEE Nuclear Science Symp. and Medical Imaging Conf. Rec., Oct. 2004, vol. 6, pp. 3997-4000.

[17] A. V. Stolin, "Characterization of imaging gamma detectors for use in small animal SPECT," in Proc. IEEE Nuclear Science Symp. and Medical Imaging Conf. Rec., Oct. 2003, vol. 3, pp. 2085-2089.

[18] E. Bradley, "A “mouse-sized" gamma camera for biological imaging," in Proc. IEEE Nuclear Science Symp. and Medical Imaging Conf. Rec., Rome, Italy, Oct. 2004.

[19] M. N. Cinti, "Iodine 125 imaging in mice using NaI(Tl)/flat panel PMT integral assembly," in Proc. IEEE Nuclear Science Symp. and Medical Imaging Conf. Rec. , Oct. 2001, vol. 6, pp. 3916-3919.

[20] E. N. Gimenez, "Detector optimization of a small animal PET camera based on continuous LSO crystals and flat panel PS-PMTs," in Proc. IEEE Nuclear Science Symp. and Medical Imaging Conf. Rec., Oct. 2004, vol. 6, pp. 3885-3889.

[21] S. Jan, "GATE: A simulation toolkit for PET and SPECT," Phys. Med. Biol., vol. 49, no. 19, pp. 4543-4561, 2004.

[22] Y. Qi, B. Tsui, B. Yoder, and E. Frey, "Characteristics of compact detectors based on pixelated $\mathrm{NaI}(\mathrm{Tl})$ crystal arrays," in Proc. IEEE Nuclear Science Symp. and Medical Imaging Conf. Rec., 2002, M10-164.

[23] R. E. Welsh, E. L. Bradley, and J. Cella, "Simultaneous 125I SPECT imaging of small animals with pinhole and parallel collimation," in Proc. IEEE Nuclear Science Symp. and Medical Imaging Conf. Rec., Oct. 2003, vol. 4, pp. 2300-2304.

[24] A. G. Weisenberger, "SPECT-CT system for small animal imaging," IEEE Trans. Nucl. Sci., vol. 50, no. 1, pp. 74-79, Feb. 2003.

[25] F. J. Beekman, "U-SPECT-I: A novel system for submillimeter-resolution tomography with radiolabelled molecules in mice," J. Nucl. Med., vol. 46, pp. 1194-1200, 2005.

[26] J. Booij, K. de Bruin, J. B. Habraken, and P. Voorn, "Imaging of dopamine transporters in rats using high resolution pinhole single photon emission tomography," Eur. J. Nucl. Med., vol. 29, pp. 1221-1224, 2002.

[27] W. P. Segars, B. M. W. Tsui, E. C. Frey, G. A. Johnson, and S. S. Berr, "Development of a 4D digital mouse phantom for molecular imaging research," Mol. Imag. Biol., vol. 5, pp. 126-127, 2003.

[28] N. Sakellios, "GATE simulations for small animal SPECT/PET using voxelized phantoms and rotating-head detectors," in Proc. IEEE Nuclear Science Symp. and Medical Imaging Conf. Rec., Oct. 2006, vol. 4, pp. 2000-2003. 